\title{
Prepuce Transillumination: A Safety Tool in Forceps-guided Circumcision
}

\author{
Maged Rihan \\ General Surgery Department, Faculty of Medicine, Cairo University, Cairo, Egypt
}

Correspondence to: Dr. Maged Rihan; email:magedrihan@hotmail.com

Received: 12 January 2020; Revised: 30 April 2020; Accepted: 03 May 2020; Available online: 27 May 2020

\begin{abstract}
Background: Injury or even amputation of the glans or the penile shaft occurs rarely but can be a tragic circumcision-related complication. The forceps-guided technique can cause this complication due to an inability to visualize the glans before incising. This study was designed to solve this problem by transilluminating the prepuce before the incision, thus exploring whether it contains any tissue between its layers and visualizing what is being done rather than performing the procedure blindly. Methods: Forceps-guided circumcision was done on 432 males, from August 2018 to July 2019. The mean age of patients was 15.57 (1-348) days. The pulled prepuce was transilluminated, showing the prepuce and verifying that no glanular or penile tissue was involved in the forceps lock before incising. Results: The mean follow-up period was 12.45 (2-35) days.
\end{abstract}

Twenty-one patients had postoperative bleeding, and six patients had a hematoma. Fourteen of the 21 patients were managed using a tight bandage; the remaining 7 patients needed surgical exploration. None of the patients had glanular or penile injury. Conclusion: Transilluminating the prepuce as a modification of forceps-guided circumcision is a protective safety step before cutting the prepuce, to eliminate the incidence of glanular or penile injuries.

Keywords: Circumcision, Prepuce, Transillumination, Forceps, Complication

Ann Afr Surg. 2021; 18(2): 75-78

DOI: http://dx.doi.org/10.4314/aas.v18i2.3

Conflicts of Interest: None

Funding: None

(C) 2021 Author. This work is licensed under the Creative Commons Attribution 4.0 International License

\section{Introduction}

Circumcision means total or partial surgical excision of the prepuce. It is commonly performed for neonates but can be done at any age $(1,2)$. Worldwide, about $25-33 \%$ of males are circumcised (3).

The rate of circumcision is $70 \%$ in USA and $6 \%$ in Britain. Circumcision for religious reasons is practiced by Jews, Muslims, Black Africans, and other racial groups. In Western communities, circumcision is commonly done for medical indications such as phimosis, paraphimosis, balanitis, and localized condylomata acuminata, or carcinoma (4-6).

Surgical techniques for circumcising are many and can be classified into any of these three groups: shield and clamp (like Plastibell, Gomco or forceps guided), dorsal slit, and excision $(5,6)$.

Complications of circumcision include bleeding, phimosis, concealed penis, infection, urinary retention, necrosis of the glans or shaft, urethrocutaneous fistulas, or iatrogenic hypospadias and epispadias (7). Injury or even amputation of the glans or the penile shaft occurs rarely but is a tragic complication. The forceps-guided technique (like using a bone-cutting forceps) is liable to 
this complication due to an inability to directly visualize the glans before incising $(3,8,9)$.

Considering these complications and problems (especially glans or shaft injuries), this study was designed to improve the outcome and solve problems that may occur by using the blind forceps-guided technique. This was done by transilluminating the prepuce before incising, thus delineating and exploring whether it contained any tissue between its layers and, accordingly, visualizing what is being done rather than performing the procedure blindly. The aim of this study is to recommend the introduction of the prepuce transillumination step before incision, as a protective step aiming to eliminate the incidence of glanular or penile injuries.

\section{Methods}

\section{Study design and population}

This prospective cohort study was conducted at the Aljedaani Hospital (a private hospital in Jeddah, Kingdom of Saudi Arabia). The Research and Ethics Review Committee at the hospital gave approval for this study. Circumcision was done for 432 males from August 2018 to July 2019. Cases were sampled randomly. The mean age of patients was 15.57 (1-348) days.

\section{Perioperative care}

A physical examination, complete blood count, and prothrombin time tests were done prior to the procedure. Cases with hypospadias or epispadias were excluded. Informed consent was obtained from the parents.

Twenty cases were done under general anesthesia (for patients more than 6 months of age) and the rest were under local anesthesia. All local anesthesia cases were discharged half an hour after the procedure and general anesthesia cases after $6 \mathrm{~h}$. All cases were seen in the outpatient clinic on the 2nd and 5th days postoperatively. Daily ibuprofen $(7 \mathrm{mg} / \mathrm{kg})$ was prescribed postoperatively. The dressing was removed by the surgeon on the 2nd day postoperative. Local application of a cream containing $0.2 \%$ nitrofurazone three times daily on the wound was recommended. All complications were reported.
Prepuce Transillumination in Forceps-guided Circumcision

\section{Surgical technique}

Skin was first prepared with betadine and draping. Next, a local anesthesia was administered using a penile dorsal nerve block by injecting the local anesthetic agent without adrenaline at a dose of $1 \mathrm{~mL}+0.1 \mathrm{~mL} / \mathrm{kg}$ body weight around the main trunk of the dorsal nerve of the penis and its ventral branch just below the symphysis pubis and deeper to the fascia on both sides of the penile suspensory ligament. The prepuce was freed from the glans by lysis of adhesions, the glans was inspected and the frenulum cut using a bipolar cautery. The edges of the prepuce were pulled out in front of the glans using a pair of small artery forceps, at the 12 and 6 o'clock positions. The bone-cutting forceps was applied across the prepuce parallel to the corona without forceful tightening and immediately in front of the glans to protect the glans, hemostasis and a platform for adequate prepuce removal flush to the forceps later on. The pulled prepuce was transilluminated using a simple light source, such as an otoscope or a light torch, held by the assistant from the back side of the prepuce without direct contact with the prepuce, after turning down the room light (Fig. 1).

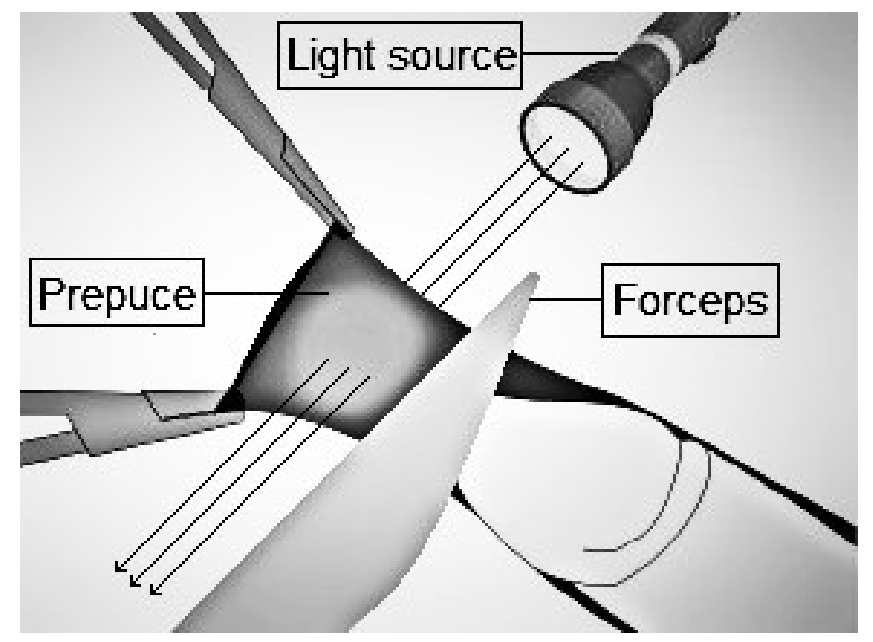

Figure 1. Transilluminating the prepuce after forceps application (courtesy of Maged Rihan)

Light is transmitted through the prepuce and illuminates and backlights it, allowing the surgeon to illustrate the prepuce and verify that no glanular or penile tissue has been caught in the lock of the forceps, in case the forceps 
are improperly placed, which appears as a dark area in the illuminated prepuce (Fig. 2).

In addition, transillumination localizes the course of blood vessels in the prepuce as a proactive step to facilitate bleeding control later on. After ensuring no other tissue is involved within the prepuce, the forceps are forcibly tightened and the scalpel is used to excise it. Hemostasis (if needed) is carried out by bipolar electrocautery. Rapid absorbable sutures (4/0 Vicryl) were used to control bleeding and to close the wound in some cases. The devices used were manufactured by Bronz Surgical Company, Jalandhar, India.

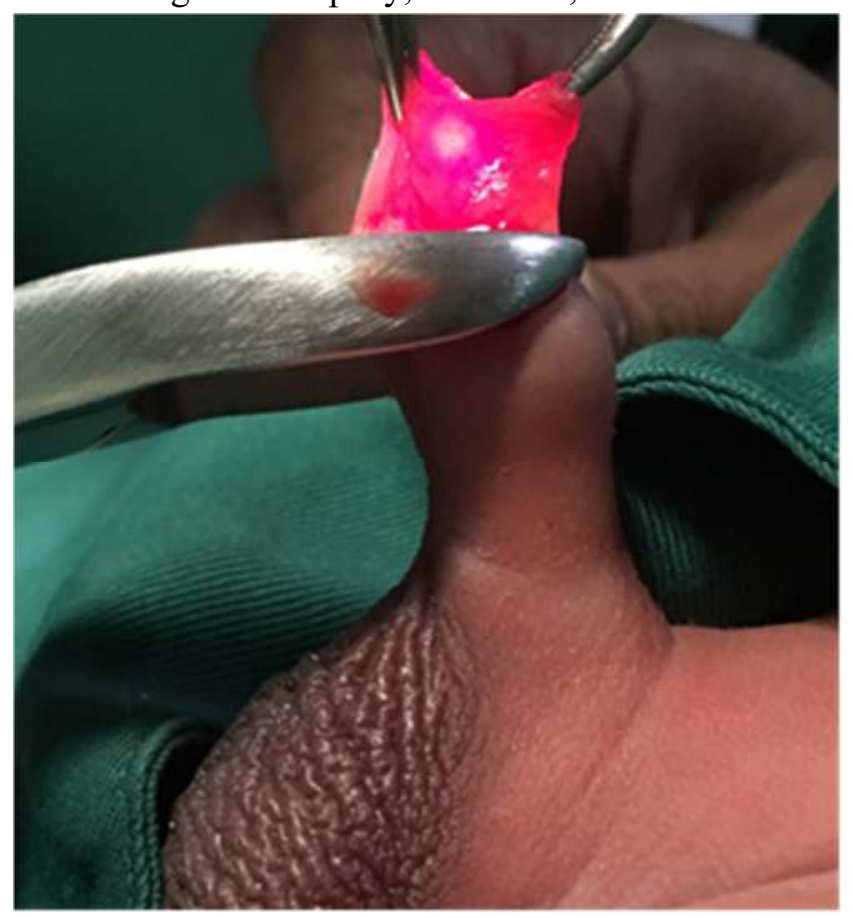

Figure 2. Light is transmitted through the shining prepuce

\section{Results}

A total of 432 males were included in the study. The mean age was 15.57 (1-348) days. The mean follow-up period was 12.45 (2-35) days. Twenty-one patients had postoperative bleeding; six patients had a hematoma. Fourteen of the 21 patients were managed using a tight bandage; the remaining 7 patients needed surgical exploration to successfully control a retracted active bleeding vessel by bipolar cautery. None of the patients had glanular or penile injury.

\section{Discussion}

Circumcision-related glanular or penile injuries have been recorded repeatedly, with varying levels of severity starting from skin or meatal injury to partial or total glanular or penile amputation. In one large series in the pediatric population, 64 boys with penile trauma were hospitalized over a period of 20 years and $43(67 \%)$ of the injuries were caused by circumcision (10). Partial removal off the glans has been recorded with the use of clamp techniques when the operator accidentally clasps the glans in the clamp (11). Circumcision by bonecutting forceps is a well-established and time-tested technique in many developing countries (12).

In a study conducted by Rehman et al. to compare the bone-cutter and open method of circumcision in which the prepuce was first fully retracted to expose the coronal sulcus and retained smegma removed, three small artery forceps were applied to the edge of the prepuce: one in the midline ventrally, two (side by side) in the midline dorsally. The prepuce was then slit in mid dorsal line between two dorsally placed artery forceps as far as the corona, taking care not to enter the urethra. The redundant part of the prepuce along with underlying inner layer were removed parallel to the corona. Hemostasis was secured with absorbable $4 / 0$ plain catgut. It was concluded that both methods proved safe and effective techniques with reproducible results, but the authors emphasized that trauma to the glans is more common with bone-cutter circumcision especially in the hands of an inexperienced operator (13).

In this study (as stated in the results), no cases were recorded of penile or glanular injuries. This may be because all the procedures were carried out by only one experienced surgeon, which may be considered as a bias in the study. It is assumed that the transillumination technique will eliminate any incidence of this type of trauma, even in less experienced hands. The aim of this study is to recommend the introduction of a prepuce transillumination step before prepuce incision as a simple and fast modification to the classical circumcision forceps-guided technique, to avoid any probability of penile or glanular injury. 


\section{Conclusion}

Circumcision is a minor operation that should be done in hospitals by trained surgeons using standard techniques such as Plastibell and Gomco, or using an open method. The use of bone-cutting forceps guided circumcision is a well-established, time tested, and widely used technique in many developing countries. Trauma to the penis including glans amputation is more frequently seen with the use of this method. Therefore, the search for cheap and fast tools to increase safety and protection is an important requirement for this technique in these countries. This study recommends the use of the transillumination step before cutting the prepuce as a modification of all forceps-guided circumcisions to eliminate the incidence of this type of trauma.

\section{References}

1. Blank S, Brady M, Buerk E, et al. American Academy of Pediatrics, Task Force on Circumcision: Male circumcision. Pediatrics. 2012;130: e756-785.

2. Morris BJ, Eley C. Male circumcision: An appraisal of current instrumentation. Chapter 14. In: Biomedical engineering: from theory to applications, Fazel-Rezai R, editor. Rijeka, Croatia: InTech, 2011:315-354.

3. Okeke LI, Asinobi AA, Ikuerowo OS. Epidemiology of complications of male circumcision in Ibadan, Nigeria. BMC Urol.2006; 6:21.

4. Gerharz EW, Haarmann C. The first cut is the deepest? Medicolegal aspects of male circumcision. BJU Int. 2000; 86:332-338.

5. Tucker SC, Cerqueiro J, Sterne GD, et al. Circumcision: A refined technique and 5-year review. Ann R Coll Surg. 2001; 83:121-125.

6. Mokal N, Chavan N. Modified safe technique for circumcision. Indian J Plast Surg. 2008; 41:47-50.

7. Ademuyiwa AO, Bode CO. Complications of neonatal circumcision: avoiding common pitfalls in a common procedure. Afr J Paediatr Surg. 2009; 6:134-136.
8. Pieretti RV, Goldstein AM, Pieretti-Vanmarcke R. Late complications of newborn circumcision: a common and avoidable problem. Pediatr Surg Int. 2010; 26:515-518.

9. Pippi Salle JL, Jesus LE, Lorenzo AJ, et al.: Glans amputation during routine neonatal circumcision: mechanism of injury and strategy for prevention. J Pediatr Urol. 2012; 9:763-768.

10. El-Bahnasawy MS, El-Sherbiny MT. Paediatric penile trauma. BJU Int. 2002;90(Suppl 1):92-96.

11. Faydaci G, Uğur K, Osman C, et al. Amputation of glans penis: A rare circumcision complication and successful management with primary anastomosis and hyperbaric oxygen therapy. Korean J Urol. 2011;52(Suppl 2):147149.

12. Amir M, Raja M, Niaz W. Neonatal circumcision with Gomco clamp - a hospital-based retrospective study of 1000 cases. J Pak Med Assoc. 2000;50(7):224-227.

13. Rehman J, Ghani MU, Shehzad K, et al. Circumcision - a comparative study. Pakistan Armed Forces Med J. 2007;57(4):286-288. 